\title{
Training Level Does Not Affect Auditory Perception of The Magnitude of Ball Spin in Table Tennis
}

\author{
by \\ Daniel P. R. Santos ${ }^{1}$, Roberto N. Barbosa', Luiz H. P. Vieira ${ }^{3}$, Paulo R. P. Santiago ${ }^{1}$, \\ Alessandro M. Zagatto ${ }^{2}$, Matheus M. Gomes ${ }^{1}$
}

\begin{abstract}
Identifying the trajectory and spin of the ball with speed and accuracy is critical for good performance in table tennis. The aim of this study was to analyze the ability of table tennis players presenting different levels of training/experience to identify the magnitude of the ball spin from the sound produced when the racket hit the ball. Four types of "forehand" contact sounds were collected in the laboratory, defined as: Fast Spin (spinning ball forward at 140 r/s); Medium Spin (105 r/s); Slow Spin (84 r/s); and Flat Hit (less than $60 \mathrm{r} / \mathrm{s}$ ). Thirty-four table tennis players of both sexes (24 men and 10 women) aged 18-40 years listened to the sounds and tried to identify the magnitude of the ball spin. The results revealed that in $50.9 \%$ of the cases the table tennis players were able to identify the ball spin and the observed number of correct answers (10.2) was significantly higher (X2 $=270.4, p<0.05)$ than the number of correct answers that could occur by chance. On the other hand, the results did not show any relationship between the level of training/experience and auditory perception of the ball spin. This indicates that auditory information contributes to identification of the magnitude of the ball spin, however, it also reveals that, in table tennis, the level of training does not interfere with the auditory perception of the ball spin.
\end{abstract}

Key words: table tennis, ball rotation, auditory information, experience level.

\section{Introduction}

Table tennis is a racket sport of the intermittent nature, which requires high speed in the production of motor responses (Padulo et al., 2016). During a match the ball can reach speeds of up to $44 \mathrm{~m} / \mathrm{s}$, with spins of up to 151 revolutions per second (r/s) (Qun et al., 1992). In addition to being considered one of the fastest sports, table tennis is also characterized as complex due to the various possibilities of strokes, combining speed, direction and rotation. The rotations of the ball upward, downward and sideways are considered to be the basic spins, and combinations thereof may generate hundreds of different spins, increasing the complexity of the motor task.

For these reasons, early awareness of the trajectory and spin of the ball could result in more time to select and decide on the most appropriate motor response, and may improve the batting in the next move. Over the years, coaches and professionals related to sports performance in table tennis have sought ways to train their athletes to anticipate identification of the ball trajectory (Ak and Koçak, 2010).

Liu (1996) indicated that the highest incidence of errors in a table tennis match was due to the difficulty of the player to identify the

\footnotetext{
1 - School of Physical Education and Sport of Ribeirão Preto, University of São Paulo, Brazil.

2 - Faculty of Sciences, São Paulo State University, Campus of Bauru, SP, Brazil.

3 - Faculty of Medicine at Ribeirão Preto, University of São Paulo, Brazil.
} 
magnitude of ball spin. The author also suggested that because of a single color of the ball, it was very difficult to identify the speed of the ball spin by means of the visual system alone. This difficulty further increased due to the high speed and large number of rotation combinations used on the ball during a hit (Hughes et al., 2002; Keele and Hawkins, 1982), resulting in a very short time for the player to identify the magnitude of the spin applied by the opponent and make a correct motor response (Liu, 1996). Therefore, early perception of the stimulus (e.g., ball spin) would result in a longer time to choose the most appropriate motor response.

In this sense, considering that the motor response after an auditory stimulus appears to be faster compared to a visual stimulus, the ability of a table tennis player to identify the spin of the ball through the contact sound of the ball with the racket could increase the chance of an optimized response. Shelton and Kumar (2010) evaluated simple reaction-time in situations with visual and auditory stimuli and found that the response to the auditory stimulus was $47 \mathrm{~ms}$ faster. Corroborating this information, Thompson et al. (1992) found that the average response time to visual stimuli was approximately 180 to $200 \mathrm{~ms}$, while for auditory stimulation it was about 140 to $160 \mathrm{~ms}$. The authors explained that the faster the stimulus reached the motor cortex, the faster the reaction time to the stimulus. Given that auditory stimuli appear to reach the motor cortex faster than visual stimuli, auditory reaction time, therefore, tends to be faster than visual reaction time. Based on this information, it is reasonable to assume that auditory perception could be used to induce faster responses in table tennis. In fact, high-performance table tennis players seem to use the sound of the contact of the ball with the racket as an indicator of the spin and speed of the ball (Bischoff et al., 2014). Bischoff et al. (2014) conducted an experiment in which the participant was required to state the flight direction of the ball from a video recording of a service in table tennis, provided or not with auditory information. The results showed that the percentage of correct predictions when given only visual information was $72.2 \%$ and when coupled with auditory information, it was $75 \%$.

According to previous findings, auditory information plays an important role in perception and action in table tennis (Bischoff et al., 2014). In addition, experience and training level are also factors that influence performance. Some studies have investigated the effects of the player experience level (i.e., advanced vs. intermediate) on the kinematic analysis of movement of the upper limbs (e.g., trunk, shoulder, elbow, forearm and wrist angular velocities) and reported that advanced players (practice time $\sim 11$ years) presented better results than intermediate players ( 7 years of practice time) (Iino and Kojima, 2009, 2011). However, the relationship between the level of training/experience in table tennis and the perception ability of table tennis players is still unknown, especially when considering the perception of auditory stimuli. Moreover, it is unclear whether auditory information when isolated from visual information is sufficient to indicate the ball spin. Therefore, some questions related to the perception of table tennis players still need to be answered. Is it possible to identify the magnitude of ball spin using only the sound produced by the contact with the racket? Is this hearing perception more accurate in table tennis players with more experience? Seeking to clarify these issues, this study aimed to analyze the ability of table tennis players, with different levels of training/experience, to identify the magnitude of ball spin from the sound produced at the instant of contact with the racket. We hypothesized that table tennis players would be able to identify the magnitude of the ball spin based on the auditory perception and that more experienced athletes would present better perception compared to the less experienced ones.

\section{Material and Methods}

\section{Participants}

The study included 34 table tennis players of both sexes ( 24 men and 10 women) with mean age of $27.00 \pm 10.37$ and $22.30 \pm 3.77$ years, body mass of $78.25 \pm 18.24 \mathrm{~kg}$ and $54.50 \pm 3.69 \mathrm{~kg}$, body height of $1.75 \pm 0.08 \mathrm{~m}$ and $1.60 \pm 0.02 \mathrm{~m}$ and training experience of $9.00 \pm 5.16$ and $11.20 \pm 6.20$ years of practice for men and women, respectively. To participate in the study, the volunteers were required to have continuously practiced table tennis for at least two years without interruption. The exclusion criteria were: the presence of any auditory dysfunction which limited sound perception or health difficulties 
that could interfere with testing. The table tennis players were invited to participate in the study in person at the 2014 South American Table Tennis Championship. After signing the informed consent form, duly approved by the Ethics Committee of the University of Sao Paulo (Protocol no. 1.055.671), the participants filled out an evaluation form containing questions about their involvement in table tennis training (e.g., number of years of training, weekly hours of training, number of competitions participated in per year) and received information on the study procedures. On average the participants presented $9.6 \pm 5.5$ years of practice; $15.8 \pm 6.8$ hours of weekly training and $10.3 \pm 5.2$ competitions per year.

\section{Procedures}

Categorization of the participants

The sample was divided into different groups according to age, gender and the level of table tennis training/experience. The groups were defined according to the following criteria: years of practice ( $<9.9$ or $\geq 10$ years); hours of weekly training ( $<15.9$ or $\geq 16$ hours / week); age ( $<20.9$ or $\geq 21$ years) and sex (male or female). The values used to categorize the participants as experienced or not were based on the study of Ericsson et al. (2007) which suggested that for an athlete to be considered experienced, more than 10 years of practice and a training load above 700 training hours per year (approximately 15 hours per week), were necessary.

Acquisition of audio files of each type of spin

The sound of each type of spin was recorded in a laboratory environment. For this purpose, a professional table tennis athlete was instructed to carry out "forehand" hits with a forward rotation (topspin), with similar velocities, but different spin magnitudes (fast, medium, slow and no spin). The balls were served to the professional table tennis player manually with a speed of approximately $5.5 \mathrm{~m} / \mathrm{s}$. In total, the table tennis player made 80 hits, aiming to perform 20 of each intensity spin. The sounds of the hits were recorded by a professional audio recorder (Roland Model R-26, Shinmiyakoda, Japan) and two microphones (Superlux model S241/U3, China) were strategically positioned in the most appropriate locations to capture audio as close as possible to the real sound.

Simultaneously with the acquisition of the audio, images of the rotations of the ball were recorded by two video cameras (EX FH25, CASIO, Tokyo, Japan) set at a frequency of acquisition of $420 \mathrm{~Hz}$ (High Speed; $224 \times 168$ pixel resolution). Half of the ball was painted red for easy viewing of ball rotations (Liu, 1996). The kinematic analysis of the ball was performed using the DVIDEOW system (Figueroa et al., 2003). The velocity was computed by videogrammetry in three dimensions. The rotation was calculated by dividing the number of frames necessary for a full rotation of the ball by the image capture frequency $(420 \mathrm{~Hz})$. In order to assess the absolute reliability of the ball rotation measurement, the standard error of measurement (SEM) was calculated by means of the following equations: SEM = Standard Deviation $\times(1$ - Intraclass Correlation Coeficient) ${ }^{0.5}$ (Gerodimos and Vassilis, 2012), from 20 analyses of ball rotation, and the SEM was $1.54 \mathrm{r} / \mathrm{s}$. For both calculations, the first frame after the contact of the ball with the racket in which the red half of the ball was displayed was considered as the initial moment. From the kinematic analysis, we selected four attempts defined as: Fast Spin (ball rotating at approximately $140 \mathrm{r} / \mathrm{s}$ ); Medium Spin (ball rotating at approximately $105 \mathrm{r} / \mathrm{s}$ ); Slow Spin (ball rotating at approximately $84 \mathrm{r} / \mathrm{s}$ ); Flat Hit (ball rotating at approximately $60 \mathrm{r} / \mathrm{s}$ ). The mean velocity for the four types of spin was between $13.6 \mathrm{~m} / \mathrm{s}$ and $14.4 \mathrm{~m} / \mathrm{s}$. Balls with similar velocities were selected in an attempt to ensure that possible differences in the recorded sounds were due to the type of spin and not the stroke speed. The audio files corresponding to each of the above attempts were used for the subsequent evaluation of auditory perception.

Picture 1 shows the organization schema of the laboratory equipment: $\mathrm{C} 1$ and $\mathrm{C} 2$ (video cameras), G1 and G2 (microphones).

Evaluation of auditory perception of ball spin

For the evaluation of auditory perception, the table tennis players were asked to identify the magnitude of ball spin from the sound caused by the contact of the ball with the racket. First the participants were informed verbally about the task they would perform and listened to 20 sounds containing the different spin types, to become familiar with the procedures. Next, five sounds of each type of spin were introduced randomly in a sequence of 20 sounds and after 
hearing every sound, the participant indicated on a record sheet the magnitude of the perceived spin (fast, medium, slow, flat). The sound transmission was generated by a laptop (Acer model Aspire, sound card Realtek ALC269 @ model Hudson-2 AMD FCH - High Definition Audio Controller, Buatan, China) and listened to through external headphones (Beats Eletronics Dr. Dre, Los Angeles, USA) adjusted to a volume of $55 \mathrm{~dB}$ in the Windows Media Player software (Windows 7, Microsoft Inc, Microsoft, Redmond, USA). The evaluations took place in a private location and lasted about 20 minutes. The number of correct answers for each type of spin was used for further comparisons.

\section{Statistical analysis}

For comparison between the levels of experience ANOVAs were conducted, given that the variables presented normality and homogeneity of variance. The independent variables for the ANOVAs were: years of practice, hours of weekly training, age and the type of spin, and the dependent variable was the number of correct answers. As the number of men and women in the categorical data was different, an ANOVA was performed with sex as an independent variable to evaluate the need to place sex as a covariate, but no difference was found between men and women. To verify that the correct answers did not occur at random, a Chi square test $\left(\mathrm{X}^{2}\right)$ was conducted, considering that the expected number of correct answers at random would be $25 \%$ ( 5 correct from a total of 20 attempts) as the possibility of answering correctly by chance was 1 in the 4 options (flat hit, slow, medium, fast spin) for each attempt. In addition, Pearson correlation tests were conducted to evaluate the relationship between the number of correct answers and the years of practice, hours of weekly training and age. For the correlation tests the independent variables were considered as continuous quantitative variables. All statistical analyzes were performed using SPSS (V17.0 - Inc. Chicago, IL, USA) and the significance level was set at 0.05 .

\section{Results}

The results revealed that in $50.9 \%$ of cases the table tennis players were able to identify the spin of the ball and the observed number of correct answers (10.2) was significantly higher $\left(X^{2}=270.4, p<0.05\right)$ than the number of correct answers that could occur by chance (5). This indicates that auditory information contributed to the identification of the magnitude of the ball spin.

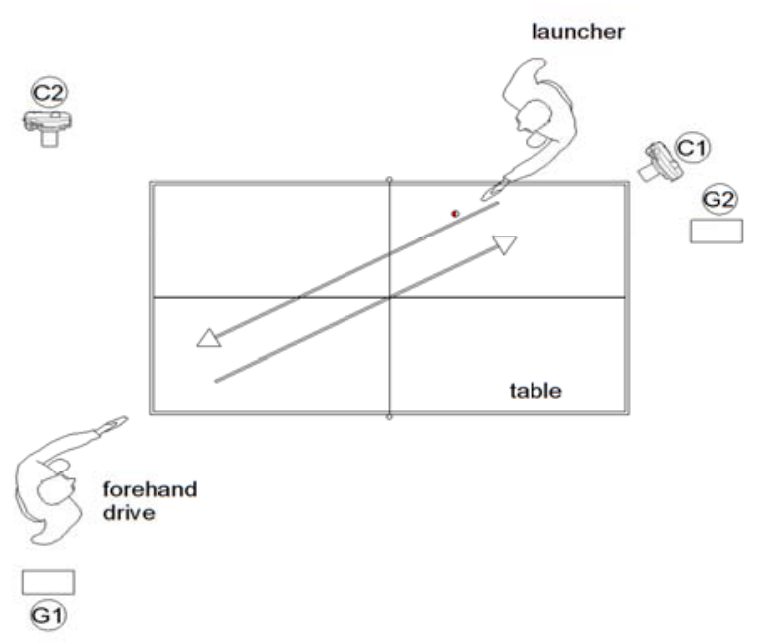

Picture 1

Organization of the equipment for the ball image and sound acquisition of the forehand stroke: C1 and C2 (video cameras), G1 and G2 (microphones). 
Table 1

Number and relative percentage of correct answers for each type of ball spin

Correct answers $\quad \%$ Correct answers

$\begin{array}{ccc}\text { Fast Spin }(140 \mathrm{r} / \mathrm{s}) & 2.1 \pm 1.6 & 42.9 \\ \text { Medium Spin }(105 \mathrm{r} / \mathrm{s}) & 2.3 \pm 1.3 & 46.5 \\ \text { Slow Spin }(84 \mathrm{r} / \mathrm{s}) & 1.8 \pm 1.3 & 36.5 \\ \text { Flat Hit }(60 \mathrm{r} / \mathrm{s})^{*} & 3.8 \pm 1.4 & 76.5 \\ \text { Total } & 10.2 \pm 3.7 & 50.9\end{array}$

* Significantly higher than other types of spin $(p<0.05)$.

Mean \pm standard deviation (SD) of the number of correct answers and the relative percentage of correct answers for each type of ball spin.

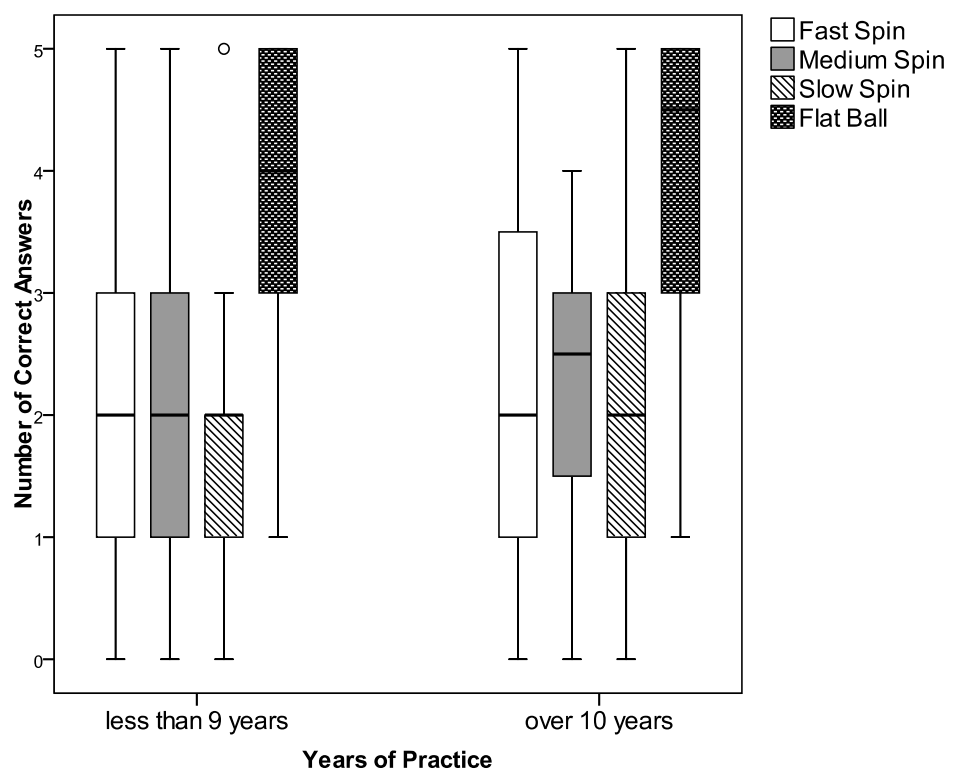

Figure 1

Distribution of correct answers of the four spin types considering the years of practice

Box plot showing the distribution of correct answers of the four spin types with the years of practice. Box plot explanation: upper horizontal line of the box, 75 th percentile; lower horizontal line of the box, 25th percentile; horizontal bar within the box, median; upper horizontal bar outside the box, maximum value; lower horizontal bar outside the box, minimum value. Circles represent outliers. 


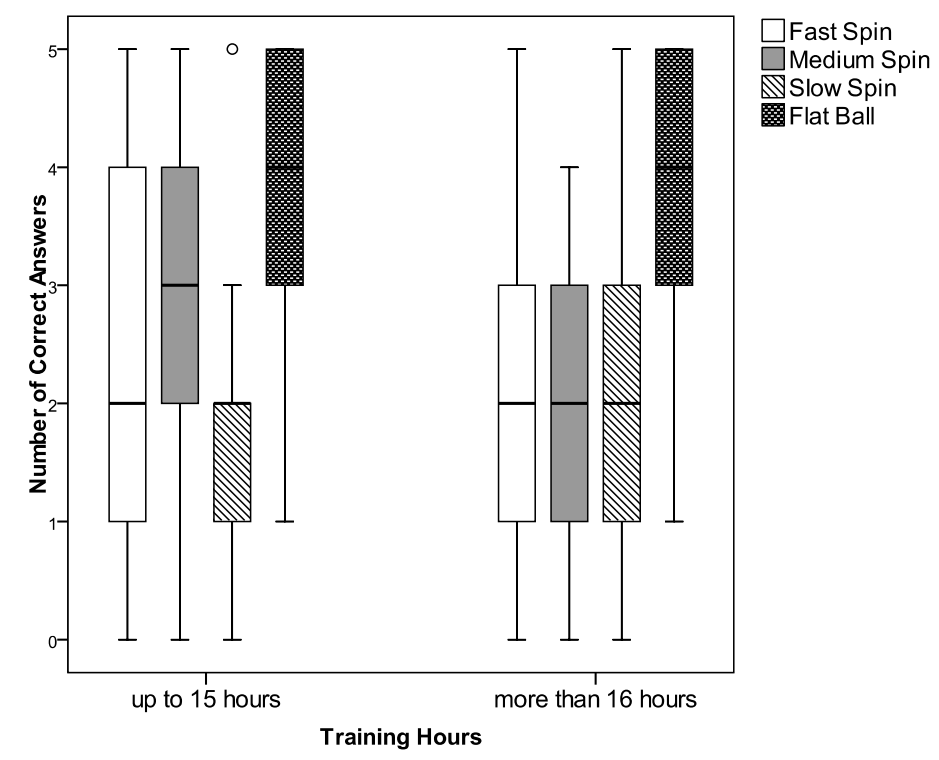

Figure 2

Distribution of correct answers of the four spin types considering the number of training hours per week

Box plot showing the distribution of correct answers of the four spin types with the training hours per week. Box plot explanation: upper horizontal line of the box, $75^{\text {th }}$ percentile; lower horizontal line of the box, 25th percentile; horizontal bar within the box, median; upper horizontal bar outside the box, maximum value; lower horizontal bar outside the box, minimum value. Circles represent outliers.

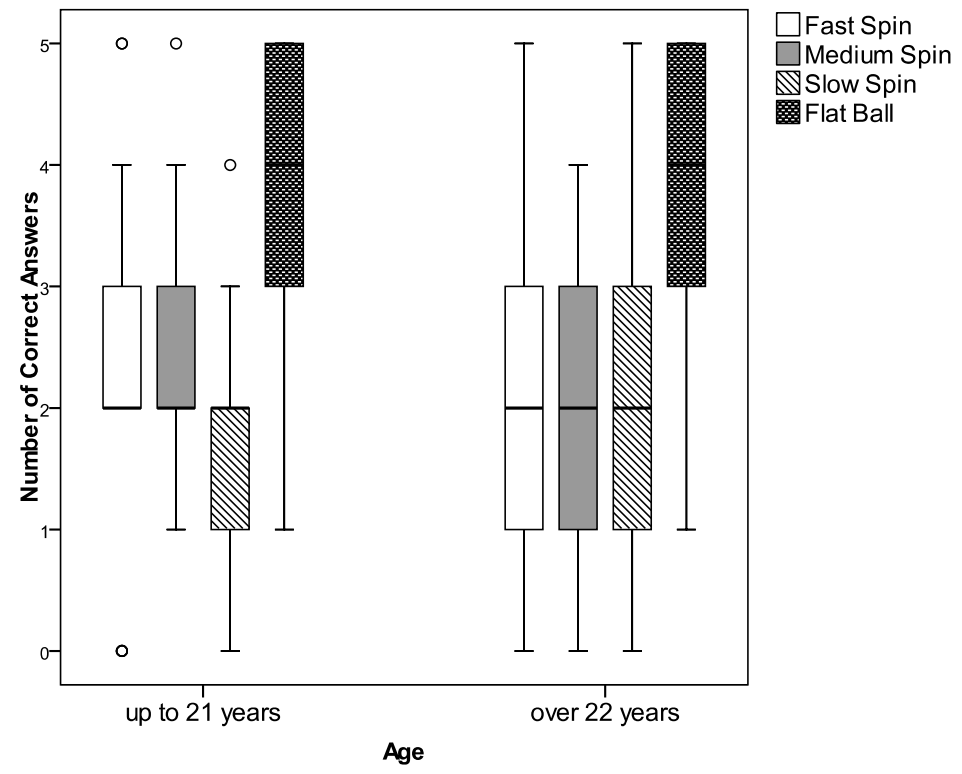

Figure 3

Distribution of correct answers of the four spin types considering age Box plot showing the distribution of correct answers of the four spin types with age.

Box plot explanation: upper horizontal line of the box, $75^{\text {th }}$ percentile; lower horizontal line of the box, 25th percentile; horizontal bar within the box, median; upper horizontal bar outside the box, maximum value; lower horizontal bar outside the box, minimum value. Circles represent outliers. 
Table 1 presents the percentage of correct answers of the different magnitudes of spin. Univariate analyzes indicated that there was a difference in the number of correct answers $\left(\mathrm{F}_{(3,132)}=13.40 ; p<0.05\right)$ and the post hoc tests revealed that the number of correct answers was greater for the flat hit compared with the other three magnitudes of spin. There was no difference in the number of correct answers in the comparisons between the fast, medium and slow spin.

Figure 1 presents the mean and standard deviation values for the total number of correct answers at the four magnitudes of spins for players with up to 9 years of practice and players with over 10 years of practice. The univariate analyses indicated no differences between years of practice $\left(\mathrm{F}_{(1,32)}=0.006 ; p>0.05\right)$. Table tennis players with more years of practice presented similar auditory perception of the ball spin to table tennis players with fewer years of practice.

Figure 2 presents the mean and standard deviation values for the total number of correct answers at the four magnitudes of spins for players who trained for up to 15 hours a week and players who trained for more than 16 hours per week. The univariate analyses indicated no differences between hours of weekly training $\left(\mathrm{F}_{(1,32)}=0.034 ; p>0.05\right)$. Table tennis players who trained for up to 15 hours a week presented similar auditory perception of the ball spin to table tennis players who trained for more than 16 hours per week.

Figure 3 presents the mean and standard deviation values for the total number of correct answers at the four magnitudes of spins for the players up to 21 years of age and players over 22 years of age. The univariate analyses indicated no differences between the two age groups $\left(\mathrm{F}_{(1,32)}=\right.$ $0.087 ; p>0.05)$. The younger table tennis players (18-21 years old) presented similar auditory perception of the ball spin to older table tennis players (over 22 years old).

\section{Discussion}

The study aimed to analyze the capability of table tennis players, with different levels of experience, to identify the magnitude of the forehand stroke ball spin through auditory perception. The results indicated that auditory perception contributed to the identification of the magnitude of ball spin, especially when a flat hit was performed. Moreover, the results showed that experience in table tennis did not influence the auditory perception of the ball spin, for being a poorly researched subject it was difficult to find related studies. We researched in Pubmed and Google Scholar platforms the following terms "table tennis perception", "table tennis spin" and "table tennis auditory information" and found 46 scientific articles, but only 10 of them related to our study. Furthermore, we also looked for such data in specific table tennis magazines as the International Journal of Table Tennis Sciences and we found several articles, but only three of them related to our study.

Perception is essential for many daily activities and audition provides perceptual information that can increase the ability to respond accurately to a stimulus (Buchtel et al., 1996). According to Desantis et al. (2014), the brain combines information from different sensory systems to respond to a stimulus, including information emitted by the auditory system. The present study demonstrated that in $50.9 \%$ of attempts, the evaluated table tennis players were able to identify the magnitude of ball spin through sound, suggesting that auditory perception can be exploited as a benefit to the athletes during matches. A more detailed analysis of the results shows that the flat hit received the highest proportion of correct answers (76.5\%), suggesting that during a match, table tennis players tend to identify balls with this intensity of rotations more easily.

In a recent study, Park et al. (2015) pointed to the contribution of auditory perception in table tennis. The authors found that after adding auditory information to visual information, the ability of table tennis players to predict the type of spin of a service improved by $15 \%$. However, the previous study presents a contradictory finding to ours, as it indicated that when the table tennis players received only auditory information (with no additional visual information), the ability to predict the direction or spin of a service was not significantly different from chance (Park et al., 2015). In contrast, our findings indicate that the number of correct answers of the sample did not occur by chance, as the number of correct answers $(10.2=50.9 \%)$ was significantly higher than the number that could occur by chance $(5=25 \%)$, 
considering that the possibility of a correct answer by chance was 1 in the 4 options (flat, slow, medium, fast) in each of the 20 attempts that the participants performed. Some factors may have contributed to the discrepancy between our results and those reported in the study by Park et al. (2015). First, the strokes used in the studies were not the same. To execute a service shot (stroke used in the previous study), the ball leaves the athlete's hand with low acceleration, almost zero, and in the vertical direction, while in a forehand shot (stroke used in the present study), the athlete returned a ball that was coming towards them in a horizontal direction at a speed of about $5.5 \mathrm{~m} / \mathrm{s}$. This difference between the strokes could cause different sounds on impact between the ball and the racket, providing distinct auditory information to the athletes. Another aspect that may have contributed is that the previous study evaluated the auditory perception with reference to three spin possibilities (topspin, sidespin or cut) and not the magnitude of the spins, as in the case of the present study, only the topspin. Moreover, it is not clear in the previous study if the speed of the ball was controlled during the serve and it seems reasonable to assume that different ball speeds could cause different sounds at the moment of contact between the ball and the racket.

Another point that could be considered is that in the study by Park et al. (2015) only experienced athletes were evaluated (over 10 years of practice in the modality). To better observe the role of experience in the auditory perception of the ball spin, the present study classified experience into different categories considering information on years of practice, age and hours of weekly training. Faber et al. (2016) stated that good performance of a table tennis athlete was directly related to the development of the learning and training experience of the player. While experience can be an important factor for performance in table tennis (Faber et al., 2016), the variables of experience evaluated in the present study were not associated with the auditory perception of table tennis players, suggesting that experience is not a decisive factor in the ability to identify the ball spin based on the sound of the hit.

However, Leontiev (1960) presented an opposite point of view, indicating that differences in the perception of sounds occurred according to the experience of each person, whether through cultural factors or activities related to the sound. The study by Antunes and Gouveia Júnior (2009) reinforces the importance of experience for auditory perception. When evaluating performance in identifying harmonic sounds in people with and without a history of contact with musical instruments, the authors found that participants who had previous contact with musical instruments performed better, indicating that in this case experience was the most important factor in the discrimination of sounds. The absence of a relationship between experience and auditory perception of the ball spin in table tennis could be related to the more commonly stimulated and/or trained sensory inputs in this sport. One hypothesis is that table tennis players principally develop and train the perception of information received from the visual and somatosensory systems. Thus, auditory perception would be little explored over the years of involvement in table tennis and hence, would not be more developed in more experienced athletes. However, this is only an assumption which could be confirmed in future studies designed to investigate the role of training in the auditory perception of table tennis players.

Importantly, our findings should be interpreted with caution due to some limitations. The results were obtained using sounds previously recorded in a laboratory environment, which may be different from the sounds heard in real game situations. Our findings were related only to the sound of the "forehand" hit forward and we do not know whether they can be generalized to other types of strokes and types of table tennis spins. In addition, the sound of the shot was presented in isolation, in a different context from a real game situation, in which image and sound complement each other.

Finally, we can conclude that table tennis players were able to satisfactorily use $50.9 \%$ of attempts) the information of the sound produced by the contact of the ball with the racket to identify the magnitude of ball spin in a forehand stroke. The correct perception of the magnitude of the spin occurred primarily when few spins were employed on the ball, as in the flat hit. However, the level of training/experience did not affect the auditory perception ability regarding the spin of 
the ball. The findings obtained in this study could help coaches and athletes in creating training methods using auditory perception to anticipate the identification of the ball spin and to have a faster reaction time in table tennis.

\section{Acknowledgements}

The authors would like to thank the Music Department of the Faculty of Philosophy, Sciences and Languages of Ribeirão Preto of the University of São Paulo for their collaboration in the acquisition of sounds of forehand strokes with different spins.

\section{References}

Ak E, Koçak S. Coincidence-anticipation timing and reaction time in youth tennis and table tennis players 1. Percept motor skills, 2010; 110: 879-887

Bischoff M, Zentgraf K, Pilgramm S, Stark R, Krüger B, Munzert J. Anticipating action effects recruits audiovisual movement representations in the ventral premotor cortex. Brain cognition, 2014; 92: 39-47

Buchtel HA, Butter CM, Ayvasik B. Effects of stimulus source and intensity on covert orientation to auditory stimuli. Neuropsychologia, 1996; 34: 979-985

Ericsson KA, Prietula MJ, Cokley ET. The making of an expert. Harvard Bus Rev, 2007; 85: 114-122

Faber IR, Elferink-Gemser MT, Faber NR, Oosterveld FG, Nijhuis-Van der Sanden MW. Can PerceptuoMotor Skills Assessment Outcomes in Young Table Tennis Players (7-11 years) Predict Future Competition Participation and Performance? An Observational Prospective Study. PLoS One, 2016; 11: $1-13$

Figueroa PJ, Leite NJ, Barros RM. A flexible software for tracking of markers used in human motion analysis. Comput Methods Programs Biomed, 2003; 72: 155-165

Gerodimos V. Reliability of handgrip strength test in basketball players. J Hum Kinet, 2012; 31: 25-36

Hughes M, Maynard I, Lees A, Reilly T. Science and Racket Sports II: Taylor \& Francis, 206-208; 2002

Iino Y, Kojima T. Kinematics of table tennis topspin forehands: effects of performance level and ball spin. J Sports Sci, 2009; 27: 1311-1321

Iino Y, Kojima T. Kinetics of the upper limb during table tennis topspin forehands in advanced and intermediate players. Sports Biomech, 2011; 10: 361-377

Keele SW, Hawkins HL. Explorations of Individual Differences Relevant to High Level Skill. J Motor Behav, 1982; 14: 3-23

Liu W. On correct spin and its control. International Joournal of Table Tennis Sciences, 1996; 3: 141-143

Padulo J, Pizzolato F, Tosi Rodrigues S, Migliaccio GM, Attene G, Curcio R, Zagatto AM. Task complexity reveals expertise of table tennis players. J Sports Med Phys Fitness, 2016; 56: 149-156

Park SH, Kim S, Kwon M, Christou EA. Differential contribution of visual and auditory information to accurately predict the direction and rotational motion of a visual stimulus. Appl Physiol Nutr Me, 2016; 41: $244-248$

Qun WH, Zhifeng Q, Shaofa X, Enting X. Experimental research in table tennis spin. International Journal of table tennis Sciences, 1992; 1: 73-78

\section{Corresponding author:}

\section{Matheus Machado Gomes}

School of Physical Education and Sport of Ribeirão Preto, University of São Paulo, Avenida Bandeirantes, 3900; Ribeirão Preto, SP, Brazil; CEP: 14049-907

Phone: +55 16 3315-0345

E-mail: mmgomes@usp.br 